\title{
On Global Stability of Disease-Free Equilibrium in Epidemiological Models
}

\author{
Tunde Tajudeen Yusuf
}

\begin{abstract}
This paper considers the problem of constructing appropriate Lyapunov function for establishing the global stability of a disease-free equilibrium in epidemiological models. A generalised algorithm is proposed and it is tested for some selected epidemiological models. Experience from the application of the algorithm on test examples shows that the algorithm is easy to use, less cumbersome, and yielded the desired result, particularly in models with homogeneous population. Thus, the proposed algorithm provides a direct approach for establishing global stability of disease-free equilibrium.
\end{abstract}

Index Terms-Disease-free equilibrium, Basic reproduction number, Lyapunov function, Lasalle's invariance principle, Global asymptotic stability.

\section{INTRODUCTION}

In mathematical Epidemiology, it is the norm to subject proposed model to qualitative analysis. An important component of the qualitative analysis is to determine the model equilibrium solutions and unveil the criteria for the stability of each of these equilibrium solutions. It is pertinent to mention that the equilibrium solutions are the possible scenarios that could emerge from the epidemiological situation being modelled while their stability helps in determining which of the scenarios will eventually play-out whenever the specified conditions are met.

Usually, epidemiological models have the disease-free equilibrium and the disease-endemic equilibrium solutions. Several authors have adopted different approaches in establishing the conditions for global stability of each of these equilibrium solutions (See [5], [9], [10]). However, majority of the authors often used the Lyapunov function approach in conjunction with LaSalle's invariance principle to establish the condition(s) for the global asymptotic stability of their model equilibrium solutions [6], [8]. Though, there seems not to be any clearly definitive approach for constructing the Lyapunov function that would yield the desired result [11]. Most times, the approach adopted in many of the research works is a " trial and error " one. Thus, making the Lyapunov function approach a cumbersome and non-definitive exercise. For instance, authors like Asamoah et al and Ullah et al used the Lyapunov function approach with different construct of the Lyapunov function candidates, though they both obtained the desired results [2], [3]. More

DOI: http://dx.doi.org/10.24018/ejmath.2021.2.3.21

Published on July 14, 2021.

T. T. Yusuf is with Federal University of Technology Akure, Ondo State, Nigeria. often than not, the relative appropriateness of the adopted Lyapunov function candidate determines the ease of showing the global stability of the equilibrium solutions.

Here, a definitive approach for constructing the Lyapunov function with respect to a disease-free equilibrium of epidemiological models is proposed. Test examples are used to illustrate that the algorithm yielded the desired result while limitations of the applications of the proposed algorithms are equally specified.

\section{Proposed Algorithm}

The proposed algorithm is as follows: $i$.

1) Given an epidemiological model, determine the model equilibriums solutions and identify which of the equilibrium solutions is the disease-free equilibrium;

2) Derive the model basis reproduction number with respect to disease under consideration using next generation matrix approach (See [4] );

3) Construct the required Lyapunov function as a linear combination of all the model infected compartments;

4) Thus, define the Lyapunov function as

$$
\mathcal{V}\left(t, S_{1}, \ldots, S_{n}, I_{1}, \ldots, I_{n}, R_{1}, \ldots, R_{n}\right)=\sum_{i=1}^{n} C_{i} I_{i} ;
$$

5) Differentiate $\mathcal{V}($.$) with respect to time to obtain$

$$
\frac{d \mathcal{V}}{d t}=\sum_{i=1}^{n} C_{i} \frac{d I_{i}}{d t}
$$

6) Substitute for each $\frac{d I_{i}}{d t}$ as defined in the model being considered;

7) Substitute the disease-free equilibrium variable value for each of the non-infected compartments in (2) to yield :

$$
\frac{d \mathcal{V}}{d t} \leq \sum_{i=1}^{n} C_{i} \frac{d I_{i}}{d t}
$$

8) Collect like terms in RHS of the resulting inequality in (3) with each of its terms expressed in terms of each of the infected compartments;

9) Choose $C_{i}$ appropriately such that the RHS in (3) has at least one of its terms expressed as a function of $\mathcal{R}_{0}$ while the other remaining terms vanish;

10) Establish that the resulting derivative of the Lyapunov function (3) is usually less than or equal zero whenever $\mathcal{R}_{0}<1$; 
11) Based on Lassalle's Invariance principle [8], conclude that the disease-free equilibrium is global asymptotically stable whenever the $\mathcal{R}_{0}<1$.

\section{TEST EXAMPLES}

\section{A. An SIR model}

Let us consider an SIR epidemiological model for the spread of Chicken pox with the availability of potent vaccine as stated below:

$$
\begin{aligned}
\frac{d S}{d t} & =\Lambda-\frac{\beta S I}{N}-(\rho+\mu) S \\
\frac{d I}{d t} & =\frac{\beta S I}{N}-(\gamma+\delta+\mu) I \\
\frac{d R}{d t} & =\rho S+\gamma I-\mu .
\end{aligned}
$$

The model ((4)) depicts the transmission dynamics of chicken pox within a variable size human population in the presence of a potent vaccine. Thus, the model divides the population of interest into three mutually exclusive compartments, namely : $S(t)$ - Susceptibles, $I(t)$ - Infectives, and $R(t)$ Recovered; with $S(t), I(t), R(t)$ representing the number of individuals in each of the respective compartments per unit time., while all the model parameter values are positive and $N(t)=S(t)+I(t)+R(t) \leq N^{*}=\frac{\Lambda}{\mu}$.

The disease-free equilibrium solution is

$$
\mathcal{E}_{1}=\left(S^{*}=\frac{\Lambda}{(\rho+\mu)}, I^{*}=0, R^{*}=\frac{\Lambda \rho}{\mu(\rho+\mu)}\right)
$$

and basic reproduction number

$$
\mathcal{R}_{0}=\frac{\beta \mu}{(\rho+\mu)(\gamma+\delta+\mu)}
$$

Theorem 3.1: The model (4) has the disease free equilibrium $\left(\mathcal{E}_{1}\right)$ whenever $\mathcal{R}_{0}<1$ and it is globally asymptotically stable (GAS).

proof 3.2: Let us consider a Lyapunov function $\mathcal{V}_{1}$ of the form below:

$$
\mathcal{V}_{1}(t, S, I, R)=C_{1} I
$$

On the differentiation of $\mathcal{V}$ with respect to $t$ in (6), we have :

$$
\begin{aligned}
\frac{d \mathcal{V}_{1}}{d t} & =C_{1} I^{\prime}, \\
& =C_{1}\left(\frac{\beta S I}{N}-(\gamma+\delta+\mu) I\right) \\
& =C_{1}\left(\frac{\beta S}{N}-(\gamma+\delta+\mu)\right) I \\
& \leq C_{1}\left(\frac{\beta \mu}{\rho+\mu}-(\gamma+\delta+\mu)\right) I \\
& \leq C_{1}(\gamma+\delta+\mu)\left(\frac{\beta \mu}{(\rho+\mu)(\gamma+\delta+\mu)}-1\right) I, \\
& \leq C_{1}(\gamma+\delta+\mu)\left(\mathcal{R}_{0}-1\right) I, \\
& \leq\left(\mathcal{R}_{0}-1\right) I, \quad C_{1}=\frac{1}{(\gamma+\delta+\mu)} .
\end{aligned}
$$

it is imperative to note that $\mathcal{V}_{1}^{\prime}=0$ only when $I=0$. Moreover, the substitution of $I=0$ into the model system of equations shows that $S \rightarrow \frac{\Lambda}{(\rho+\mu)}$ and $R \rightarrow \frac{\Lambda \rho}{\mu(\rho+\mu)}$ as $t \rightarrow \infty$. Based on LaSalle's invariance principle [8], $\mathcal{E}_{1}$ is globally asymptotically stable whenever $\mathcal{R}_{0}<1$.

\section{B. Model for Meningitis disease transmission dynamics}

Consider the model for the spread of Meningococcal Meningitis disease below:

$$
\begin{aligned}
\frac{d S}{d t} & =\Lambda-\frac{\beta S\left(I_{1}+I_{2}\right)}{N}-\left(u_{1}+\mu\right) S+\sigma R \\
\frac{d I_{1}}{d t} & =\frac{\beta S\left(I_{1}+I_{2}\right)}{N}-(\rho+\gamma+\mu) I_{1} \\
\frac{d I_{2}}{d t} & =\rho I_{1}-\left(u_{2}+\mu+\delta\right) I_{2} \\
\frac{d R}{d t} & =u_{1} S+\gamma I_{1}+u_{2} I_{2}-(\sigma+\mu) R
\end{aligned}
$$

It salient to mention that the model (8) represents the dynamics of the spread of Meningitis disease among human population where effective treatment given to patients and vaccination of the susceptibles only confer temporary immunity. Hence, this model divides the population of interest into four mutually exclusive compartments, namely : $S(t)$ - Susceptibles, $I_{1}(t)$ Carriers, $I_{2}(t)$ - Infected individuals, and $R(t)$ - Recovered individuals; with $S(t), I_{1}(t), I_{2}(t), R(t)$ representing the number of individuals in each of the respective compartments per unit time., while all the model parameter values are positive and $N(t)=S(t)+I_{1}(t)+I_{2}(t)+R(t)$.

In this case, both disease compartments $I_{1}(t)$, and $I_{2}(t)$ are infectious while the model disease-free equilibrium $\left(\mathcal{E}_{2}\right)$ and basic reproduction number $\left(\mathcal{R}_{0}\right)$ are as respectively stated below:

The disease-free equilibrium solution:

$\mathcal{E}_{2}=\left(S^{*}=\frac{\Lambda(\sigma+\mu)}{\mu\left(u_{1}+\sigma+\mu\right)}, I_{1}^{*}=0, I_{2}^{*}=0, R^{*}=\frac{\Lambda u_{1}}{\mu\left(u_{1}+\sigma+\mu\right)}\right)$

and basic reproduction number

$\mathcal{R}_{0}=\frac{\beta(\sigma+\mu)}{\left(u_{1}+\sigma+\mu\right)(\rho+\gamma+\mu)}+\frac{\beta(\sigma+\mu) \rho}{\left(u_{1}+\sigma+\mu\right)(\rho+\gamma+\mu)\left(u_{2}+\mu+\delta\right)}$

So, in order to show that the model disease-free equilibrium is GAS, we adopt the proposed algorithm as below:

Theorem 3.3: The model ((8)) has the disease free equilibrium $\left(\mathcal{E}_{2}\right)$ whenever $\mathcal{R}_{0}<1$ and it is globally asymptotically stable (GAS).

proof 3.4: Suppose $\mathcal{R}_{0}<1$, then the model has only the disease-free equilibrium $\left(\mathcal{E}_{2}\right)$ as its only equilibrium. So, we only need to show that $\left(\mathcal{E}_{2}\right)$ is GAS. Let us consider a Lyapunov function $\mathcal{V}_{2}$ of the form below:

$$
\mathcal{V}_{2}\left(t, S, I_{1}, I_{2}, R\right)=C_{1} I_{1}+C_{2} I_{2} .
$$


On the differentiation of $\mathcal{V}_{2}$ with respect to $\mathrm{t}$ in (10), we have:

$$
\begin{aligned}
\frac{d \mathcal{V}_{2}}{d t} & =C_{1} I_{1}^{\prime}+C_{2} I_{2}^{\prime} \\
& =C_{1}\left(\frac{\beta S\left(I_{1}+I_{2}\right)}{N}-(\rho+\gamma+\mu) I_{1}\right) \\
& +C_{2}\left(\rho I_{1}-\left(u_{2}+\mu+\delta\right) I_{2}\right), \\
& =\left(\frac{C_{1} \beta S}{N}+C_{2} \rho-C_{1}(\rho+\gamma+\mu)\right) I_{1} \\
& +\left(\frac{C_{1} \beta S}{N}-C_{2}\left(u_{2}+\mu+\delta\right)\right) I_{2}, \\
\leq & \left(\frac{C_{1} \beta(\sigma+\mu)}{u_{1}+\sigma+\mu}+C_{2} \rho-C_{1}(\rho+\gamma+\mu)\right) I_{1} \\
& +\left(\frac{C_{1} \beta(\sigma+\mu)}{u_{1}+\sigma+\mu}-C_{2}\left(u_{2}+\mu+\delta\right)\right) I_{2}
\end{aligned}
$$

Taking $S=S^{*}=\frac{\Lambda(\sigma+\mu)}{\mu\left(u_{1}+\sigma+\mu\right)}, \quad N=N^{*}=\frac{\Lambda}{\mu}$,

$$
\begin{aligned}
& \leq\left(\frac{\beta(\sigma+\mu)}{u_{1}+\sigma+\mu}+\frac{\beta \rho(\sigma+\mu)}{\left(u_{1}+\sigma+\mu\right)\left(u_{2}+\mu+\delta\right)}-(\rho+\gamma+\mu)\right) I_{1} \\
& +\left(\frac{\beta(\sigma+\mu)}{u_{1}+\sigma+\mu}-\frac{\beta(\sigma+\mu)}{u_{1}+\sigma+\mu}\right) I_{2}
\end{aligned}
$$$$
\text { where } C_{1}=1, C_{2}=\frac{\beta(\sigma+\mu)}{\left(u_{1}+\sigma+\mu\right)\left(u_{2}+\mu+\delta\right)} \text {, }
$$$$
\leq(\rho+\gamma+\mu)\left(\frac{\beta(\sigma+\mu)}{\left(u_{1}+\sigma+\mu\right)(\rho+\gamma+\mu)}\right) I_{1}+
$$$$
(\rho+\gamma+\mu)\left(\frac{\beta \rho(\sigma+\mu)}{(\rho+\gamma+\mu)\left(u_{1}+\sigma+\mu\right)\left(u_{2}+\mu+\delta\right)}-1\right) I_{1},
$$$$
\leq(\rho+\gamma+\mu)\left(\mathcal{R}_{0}-1\right) I_{1} .
$$

In view of the foregoing, it is imperative to note that $\mathcal{V}_{2}^{\prime}=0$ only when $I_{1}=0$. Moreover, the substitution of $I_{1}=0$, and $I_{2}=0$ into the model system of equations gives $S \rightarrow \frac{\Lambda(\sigma+\mu)}{\mu\left(u_{1}+\sigma+\mu\right)}$ and $R \rightarrow \frac{\Lambda u_{1}}{\mu\left(u_{1}+\sigma+\mu\right)}$ as $t \rightarrow \infty$. Based on LaSalle's invariance principle [8], $\mathcal{E}_{2}$ is globally asymptotically stable whenever $\mathcal{R}_{0}<1 \square$

\section{Model for Hepatitis B Virus (HBV) disease transmission dynamics [7]}

Consider the model for the spread of HBV disease below:

$$
\begin{aligned}
\frac{d S}{d t} & =\Lambda-\frac{\beta S I_{1}}{N}-\frac{\beta S I_{2}}{N}-(\nu+\mu) S \\
\frac{d I_{1}}{d t} & =\frac{\beta S I_{1}}{N}+\frac{\beta S I_{2}}{N}-\left(\rho_{1}+\gamma+\mu\right) I_{1} \\
\frac{d I_{2}}{d t} & =\rho_{1} I_{1}-\left(\rho_{2}+\mu\right) I_{2} \\
\frac{d I_{3}}{d t} & =\rho_{2} I_{2}-(\mu+\delta) I_{3} \\
\frac{d R}{d t} & =\nu S+\gamma I_{1}-\mu R
\end{aligned}
$$

It is important to note that this model ((12)) captures the dynamical spread of HBV disease within the human population in a situation where there is effective vaccine which confers permanent immunity on recipients. So, the model divides the population of interest into four mutually exclusive compartments, namely : $S(t)$ - Susceptibles, $I_{1}(t)$ - Exposed individuals, $I_{2}(t)$ - Acute Infected individuals, $I_{3}(t)$ - Chronic Infected individuals, and $R(t)$ - Recovered individuals; with $S(t), I_{1}(t), I_{2}(t), R(t)$ representing the number of individuals in each of the respective compartments per unit time., while all the model parameter values are positive and $N(t)=S(t)+I_{1}(t)+I_{2}(t)+I_{3}(t)+R(t)$.

In this case, the Exposed compartment $I_{1}(t)$ is not infectious while the other two disease compartments $I_{2}(t)$, and $I_{3}(t)$ are both infectious while the model disease-free equilibrium $\left(\mathcal{E}_{3}\right)$ and basic reproduction number $\left(\mathcal{R}_{0}\right)$ are as respectively stated below:

The disease-free equilibrium solution:

$\mathcal{E}_{3}=\left(S^{*}=\frac{\Lambda}{(\nu+\mu)}, I_{1}^{*}=0, I_{2}^{*}=0, I_{3}^{*}=0, R^{*}=\frac{\Lambda \nu}{\mu(\nu+\mu)}\right) ;$ and basic reproduction number

$\mathcal{R}_{0}=\frac{\beta_{1} \rho_{1} \mu}{(\nu+\mu)\left(\rho_{1}+\gamma+\mu\right)\left(\rho_{2}+\mu\right)}+\frac{\beta_{2} \rho_{1} \rho_{2} \mu}{(\nu+\mu)\left(\rho_{1}+\gamma+\mu\right)(\delta+\mu)\left(\rho_{2}+\mu\right)}$

So, in order to show that the model disease-free equilibrium is GAS, we adopt the proposed algorithm as below:

Theorem 3.5: The model 12 has the disease free equilibrium $\left(\mathcal{E}_{3}\right)$ whenever $\mathcal{R}_{0}<1$ and it is globally asymptotically stable (GAS).

proof 3.6: Suppose $\mathcal{R}_{0}<1$, then the model has only the disease-free equilibrium $\left(\mathcal{E}_{3}\right)$ as its only equilibrium. So, we only need to show that $\left(\mathcal{E}_{3}\right)$ is GAS. Let us consider a Lyapunov function $\mathcal{V}_{3}$ of the form below:

$$
\mathcal{V}_{3}\left(t, S, I_{1}, I_{2}, I_{3}, R\right)=C_{1} I_{1}+C_{2} I_{2}+C_{3} I_{3} .
$$

On the differentiation of $\mathcal{V}_{3}$ with respect to $t$ in (13), we have

$$
\begin{aligned}
\frac{d \mathcal{V}_{3}}{d t=} & C_{1} I_{1}^{\prime}+C_{2} I_{2}^{\prime}+C_{3} I_{3}^{\prime}, \\
= & C_{1}\left(\frac{\beta_{1} S I_{2}}{N}+\frac{\beta_{2} S I_{3}}{N}-\left(\gamma+\rho_{1}+\mu\right) I_{1}\right) \\
& +C_{2}\left(\rho_{1} I_{1}-\left(\rho_{2}+\mu\right) I_{2}\right)+C_{3}\left(\rho_{2} I_{2}-(\mu+\delta) I_{3}\right), \\
\leq & C_{1}\left(\frac{\beta_{1} \mu I_{2}}{\nu+\mu}+\frac{\beta_{2} \mu I_{3}}{\nu+\mu}-\left(\gamma+\rho_{1}+\mu\right) I_{1}\right. \\
& +C_{2}\left(\rho_{1} I_{1}-\left(\rho_{2}+\mu\right) I_{2}\right)+C_{3}\left(\rho_{2} I_{2}-(\mu+\delta) I_{3}\right) .
\end{aligned}
$$

Re-arranging the preceding inequality (14) in terms of $I_{1}, I_{2}$, and $I_{3}$, we obtain

$$
\begin{aligned}
\frac{d \mathcal{V}_{3}}{d t} & \leq\left(C_{2} \rho_{1}-C_{1}\left(\gamma+\rho_{1}+\mu\right)\right) I_{1} \\
& +\left(\frac{C_{1} \beta_{1} \mu}{\nu+\mu}+C_{3} \rho_{2}-C_{2}\left(\rho_{2}+\mu\right)\right) I_{2}+ \\
& \left(\frac{C_{1} \beta_{2} \mu}{\nu+\mu}-C_{3}(\mu+\delta)\right) I_{3} .
\end{aligned}
$$

Thus, substituting $C_{1}=\frac{\rho_{1}}{\gamma+\rho_{1}+\mu}, C_{2}=1$, and $\frac{\beta_{2} \mu \rho_{1}}{(\nu+\mu)(\mu+\delta)\left(\gamma+\rho_{1}+\mu\right)}$ into (15) yields : 


$$
\begin{aligned}
\frac{d \mathcal{V}_{3}}{d t} & \leq\left(\rho_{1}-\left(\gamma+\rho_{1}+\mu\right) \cdot \frac{\rho_{1}}{\gamma+\rho_{1}+\mu}\right) I_{1} \\
& +\left(\frac{\beta_{1} \mu \rho_{1}}{(\nu+\mu)\left(\gamma+\rho_{1}+\mu\right)}+\frac{\beta_{2} \mu \rho_{1} \rho_{2}}{(\nu+\mu)(\mu+\delta)\left(\gamma+\rho_{1}+\mu\right)}\right) I_{2} \\
& -\left(\rho_{2}+\mu\right) I_{2} \\
& +\left(\frac{\beta_{2} \mu \rho_{1}}{(\nu+\mu)\left(\gamma+\rho_{1}+\mu\right)}-\frac{\beta_{2} \mu \rho_{1}(\mu+\delta)}{(\nu+\mu)(\mu+\delta)\left(\gamma+\rho_{1}+\mu\right)}\right) I_{3}, \\
& \leq\left(\rho_{2}+\mu\right)\left(\mathcal{R}_{0}-1\right) I_{2}
\end{aligned}
$$

Based on the inequality (16), it is worthy to note that $\mathcal{V}_{3}^{\prime}=0$ only when $I_{2}=0$. Also, the substitution of $I_{1}=0, I_{2}=0$ and $I_{3}=0$ into the model system of equations makes $S \rightarrow \frac{\Lambda}{(\nu+\mu)}$ and $R \rightarrow \frac{\Lambda \nu}{\mu(\nu+\mu)}$ as $t \rightarrow \infty$. So, $\mathcal{E}_{3}$ is globally asymptotically stable whenever $\mathcal{R}_{0}<1$; premised on LaSalle's invariance principle [8].

\section{Model for Coronavirus disease Transmission dynamics}

Let us consider the Covid-19 disease transmission model as given below:

$$
\begin{aligned}
\frac{d S}{d t} & =\Lambda+\omega R-\left(1-u_{1}\right)\left(\beta_{1} I_{1}+\beta_{2} I_{3}+\beta_{3} I_{2}\right) S-\mu S, \\
\frac{d I_{1}}{d t} & =\left(1-u_{1}\right)\left(\beta_{1} I_{1}+\beta_{2} I_{3}+\beta_{3} I_{2}\right) S-u_{2} I_{1}-\rho I_{1}-\mu I_{1}, \\
\frac{d I_{2}}{d t} & =\rho I_{1}-u_{3} I_{2}-\gamma I_{2}-\left(\mu+\delta_{2}\right) I_{2}, \\
\frac{d I_{3}}{d t} & =u_{2} I_{1}+u_{3} I_{2}-u_{4} I_{3}-\left(\mu+\delta_{1}\right) I_{3}, \\
\frac{d R}{d t} & =\gamma I_{2}+u_{4} I_{3}-\omega R-\mu R
\end{aligned}
$$

It worth mentioning that the model ((17)) describes the dynamics of Covid-19 disease spread with the impacts of the nonpharmaceutical control measures. So, the human population under consideration is divided into five mutually exclusive compartments, namely Susceptible class $S(t)$, Asymptotically Infected $I_{1}(t)$, asymptotically Infected $I_{2}(t)$, Hospitalized under isolation $I_{3}(t)$, and Recovered $R(t)$ compartments while all the model parameters are positive. Note, for this disease compartments $I_{1}(t), I_{2}(t)$, and $I_{3}(t)$ are all infectious while the model disease-free equilibrium $\left(\mathcal{E}_{3}\right)$ and basic reproduction number $\left(\mathcal{R}_{0}\right)$ are as respectively state below:

The disease-free equilibrium solution:

$$
\mathcal{E}_{4}=\left(S^{*}=\frac{\Lambda}{\mu}, I_{1}^{*}=0, I_{2}^{*}=0, I_{3}^{*}=0, R^{*}=0\right) ;
$$

and basic reproduction number

$$
\mathcal{R}_{0}=\frac{\Lambda\left(1-u_{1}\right)}{\mu}\left(\frac{\beta_{1}}{x}+\frac{\beta_{2}\left(\rho u_{3}+y u_{2}\right)}{x y z}+\frac{\beta_{3} \rho}{x y}\right) .
$$

where $x=\mu+\rho+u_{2} ; y=\mu+\gamma+\delta_{2}+u_{3} ; z=\mu+\delta_{1}+u_{4}$.
Theorem 3.7: The model (17) has the disease free equilibrium $\left(\mathcal{E}_{4}\right)$ as its only equilibrium solution whenever $\mathcal{R}_{0}<1$ and it is globally asymptotically stable (GAS).

proof 3.8: Suppose $\mathcal{R}_{0}<1$, then the model has only the disease-free equilibrium $\left(\mathcal{E}_{4}\right)$ as its only equilibrium. So, we only need to show that $\left(\mathcal{E}_{4}\right)$ is GAS. Let us consider a Lyapunov function $\mathcal{V}_{4}$ of the form below:

$$
\mathcal{V}_{4}\left(t, S, I_{1}, I_{2}, I_{3}, R\right)=C_{1} I_{1}+C_{2} I_{2}+C_{3} I_{3}
$$

On the differentiation of $\mathcal{V}$ with respect to $\mathrm{t}$ in (18), we have:

$$
\begin{aligned}
\frac{d \mathcal{V}_{4}}{d t}= & C_{1} I_{1}^{\prime}+C_{2} I_{2}^{\prime}+C_{3} I_{3}^{\prime} \\
= & C_{1}\left(\left(1-u_{1}\right)\left(\beta_{1} I_{1}+\beta_{2} I_{3}+\beta_{3} I_{2}\right) S-u_{2} I_{1}-\rho I_{1}-\mu I_{1}\right) \\
& +C_{2}\left(\rho I_{1}-u_{3} I_{2}-\gamma I_{2}-\left(\mu+\delta_{2}\right) I_{2}\right) \\
& +C_{3}\left(u_{2} I_{1}+u_{3} I_{2}-u_{4} I_{3}-\left(\mu+\delta_{1}\right) I_{3}\right), \\
\leq & C_{1}\left(\left(1-u_{1}\right)\left(\beta_{1} I_{1}+\beta_{2} I_{3}+\beta_{3} I_{2}\right)\left(\frac{\Lambda}{\mu}\right)-u_{2} I_{1}-\rho I_{1}-\mu I_{1}\right) \\
& +C_{2}\left(\rho I_{1}-u_{3} I_{2}-\gamma I_{2}-\left(\mu+\delta_{2}\right) I_{2}\right) \\
& +C_{3}\left(u_{2} I_{1}+u_{3} I_{2}-u_{4} I_{3}-\left(\mu+\delta_{1}\right) I_{3}\right) ; \\
& \text { Taking } S=S^{*}=\frac{\Lambda}{\mu}, \\
\leq & \left(C_{1}\left(1-u_{1}\right)\left(\frac{\Lambda}{\mu}\right) \beta_{1}+C_{2} \rho+C_{3} u_{2}-C_{1}\left(\mu+\rho+u_{2}\right)\right) I_{1} \\
& +\left(C_{1}\left(1-u_{1}\right)\left(\frac{\Lambda}{\mu}\right) \beta_{2}-C_{3}\left(\mu+u_{4}+\delta_{1}\right)\right) I_{3} \\
& +\left(C_{1}\left(1-u_{1}\right)\left(\frac{\Lambda}{\mu}\right) \beta_{3}+C_{3} u_{3}-C_{2}\left(\mu+\gamma+u_{3}+\delta_{2}\right)\right) I_{2}, \\
\leq & \left(\mathcal{R}_{0}-1\right) I_{1}, \\
& \text { where } C_{1}=1, C_{2}=\frac{\Lambda\left(1-u_{1}\right)\left(\beta_{3}\left(u_{4}+\delta_{1}+\mu\right)+\beta_{2} u_{3}\right)}{\mu\left(\mu+u_{4}+\delta_{1}\right)\left(\mu+\gamma+u_{3}+\delta_{2}\right)}, \\
& \text { and } C_{3}=\frac{\Lambda\left(1-u_{1}\right) \beta_{2}}{\mu\left(\mu+u_{4}+\delta_{1}\right)}
\end{aligned}
$$

Thus, it is imperative to note that $\mathcal{V}_{4}^{\prime}=0$ only when $I_{1}=0$. Moreover, the substitution of $I_{1}=0, I_{2}=0, I_{3}=$ 0 , and $R=0$ into the model system of equations indicates that $S \rightarrow \frac{\Lambda}{\mu}$ as $t \rightarrow \infty$. Based on LaSalle's invariance principle [8], $\mathcal{E}_{4}$ is globally asymptotically stable whenever $\mathcal{R}_{0}<1$.

\section{E. Model for HIV and HBV Co-epidemics [12]}

Consider a model for HIV and HBV co-epidemics in population subdivided into four mutually exclusive compartments, namely Susceptible class $S(t)$, the HBV Infected only class $I_{B}(t)$, HIV infected only class $I_{H}(t)$, and the HIV/HBV co-infected class $I_{C}(t)$ while all the model parameters are positive. The dynamics of the spread of the two diseases is as 
given below:

$$
\begin{aligned}
\frac{d S}{d t} & =\Pi-\gamma\left(I_{B}+I_{C}\right) \frac{S}{N}-\tau\left(I_{H}+I_{C}\right) \frac{S}{N}-\mu S \\
\frac{d I_{B}}{d t} & =\gamma\left(I_{B}+I_{C}\right) \frac{S}{N}-\tau\left(I_{H}+I_{C}\right) \frac{I_{B}}{N}-\left(\mu+\mu_{B}\right) I_{B}, \\
\frac{d I_{H}}{d t} & =\tau\left(I_{H}+I_{C}\right) \frac{S}{N}-\gamma\left(I_{B}+I_{C}\right) \frac{I_{H}}{N}-\left(\mu+\mu_{H}\right) I_{H}, \\
\frac{d I_{C}}{d t} & =\gamma\left(I_{B}+I_{C}\right) \frac{I_{H}}{N}+\tau\left(I_{H}+I_{C}\right) \frac{I_{B}}{N}-\left(\mu+\mu_{B}+\mu_{H}\right) I_{C}
\end{aligned}
$$

Here, the model (20) describes the dynamics of the spread of two diseases (HIV and HBV) which share a common transmission routes; thus raising the likelihood of individuals getting infected with the two diseases. Considering the fact that HIV and HBV have no permanent cure for now, the continuous co-infection of individuals with the two diseases could eventually result into a co-epidemics if timely and effective control measures are not put in place.

Theorem 3.9: The disease free equilibrium $\mathcal{E}_{5}=\left(\frac{\Pi}{\mu}, 0,0,0\right)$ is globally asymptotically stable, whenever $\mathcal{R}_{0}<1$.

proof 3.10: Let us consider a Lyapunov function of the form.

$$
\mathcal{V}_{5}\left(S, I_{B}, I_{H}, I_{C}\right)=C_{1} I_{B}+C_{2} I_{H}+C_{3} I_{C}
$$

Differentiating $\mathcal{V}_{5}\left(S, I_{B}, I_{H}, I_{C}\right)$ with respect to $t$ gives:

$$
\frac{d \mathcal{V}_{5}}{d t}=C_{1} I_{B}^{\prime}+C_{2} I_{H}^{\prime}+C_{3} I_{C}^{\prime}
$$

Substituting for $I_{B}^{\prime}, I_{H}^{\prime}$, and $I_{C}^{\prime}$ in equation (22) yields

$$
\begin{aligned}
\frac{d \mathcal{V}_{5}}{d t}= & C_{1}\left[\gamma\left(I_{B}+I_{C}\right) \frac{S}{N}-\tau\left(I_{H}+I_{C}\right) \frac{I_{B}}{N}-\left(\mu+\mu_{B}\right) I_{B}\right] \\
& +C_{2}\left[\tau\left(I_{H}+I_{C}\right) \frac{S}{N}-\gamma\left(I_{B}+I_{C}\right) \frac{I_{H}}{N}-\left(\mu+\mu_{H}\right) I_{H}\right] \\
& +C_{3}\left[\gamma\left(I_{B}+I_{C}\right) \frac{I_{H}}{N}+\tau\left(I_{H}+I_{C}\right) \frac{I_{B}}{N}\right. \\
& \left.\left.-\left(\mu+\mu_{B}+\mu_{H}\right) I_{C}\right)\right]
\end{aligned}
$$

At the disease free equilibrium $\mathcal{E}_{5}=\left[\frac{\Pi}{\mu}, 0,0,0\right]$, with $\mathcal{R}_{0}=\max \left[\mathcal{R}_{B}=\frac{\gamma}{\mu+\mu_{B}}, \mathcal{R}_{H}=\frac{\tau}{\mu+\mu_{H}}\right]$, while $\frac{\gamma}{\mu+\mu_{B}+\mu_{H}}<$ $\frac{\gamma}{\mu+\mu_{B}}$, and $\frac{\tau}{\mu+\mu_{B}+\mu_{H}}<\frac{\tau}{\mu+\mu_{H}}$; equation (23) can be simplified as below :

$$
\begin{aligned}
\frac{d \mathcal{V}_{5}}{d t} \leq & C_{1}\left[\gamma I_{B}+\gamma I_{C}-\left(\mu+\mu_{B}\right) I_{B}\right] \\
& +C_{2}\left[\tau I_{H}+\tau I_{C}-\left(\mu+\mu_{H}\right) I_{H}\right] \\
& -C_{3}\left[\mu+\mu_{B}+\mu_{H}\right] I_{C} \\
\leq & C_{1}\left[\gamma-\left(\mu+\mu_{B}\right)\right] I_{B}+C_{2}\left[\tau-\left(\mu+\mu_{H}\right)\right] I_{H} \\
+ & {\left[C_{1} \gamma+C_{2} \tau-C_{3}\left(\mu+\mu_{B}+\mu_{H}\right)\right] I_{C}, } \\
\leq & C_{1}\left(\mu+\mu_{B}\right)\left[\mathcal{R}_{B}-1\right] I_{B}+C_{2}\left(\mu+\mu_{H}\right)\left[\mathcal{R}_{H}-1\right] I_{H} \\
& \left(\mu+\mu_{B}+\mu_{H}\right)\left[\frac{C_{1} \gamma}{\mu+\mu_{B}+\mu_{H}}+\frac{C_{2} \tau}{\mu+\mu_{B}+\mu_{H}}-C_{3}\right] I_{C}
\end{aligned}
$$

Substituting $C_{1}=C_{2}=1, C_{3}=\frac{\gamma+\tau}{\mu+\mu_{B}+\mu_{H}}$ and $\mathcal{R}_{0}=$ $\max \left[\mathcal{R}_{B}=\frac{\gamma}{\mu+\mu_{B}}, \mathcal{R}_{H}=\frac{\gamma}{\mu+\mu_{H}}\right]$ in (24) yields

$$
\begin{aligned}
\frac{d \mathcal{V}_{5}}{d t} & \leq\left(\mu+\mu_{B}\right)\left[\mathcal{R}_{0}-1\right] I_{B}+\left(\mu+\mu_{H}\right)\left[\mathcal{R}_{0}-1\right] I_{H}, \\
& \leq 0 .
\end{aligned}
$$

It is important to note that $\mathcal{V}_{5}^{\prime}=0$ only at the disease-free equilibrium $\mathcal{E}_{4}$, otherwise it is negative. Therefore, it follows from Lasalle's invariance principle that all solutions of the model equations converges to the $\mathcal{E}_{5}$ as $t \rightarrow \infty$ whenever $\mathcal{R}_{0}<1$. Hence, $\mathcal{E}_{5}$ is globally asymptotically stable whenever $\mathcal{R}_{0}<1$

\section{CONCLUding REMARKS}

In this paper, an algorithm for establishing the global stability of a disease-free equilibrium with respect to epidemiological models is developed. The algorithm is tested using some selected epidemiological models. The algorithm works perfectly for the series of models considered. Based on the experience from the test examples, it can be concluded that the proposed algorithm works fine for the purpose for which it was designed. It is pretty easy to use and understand with minimal ambiguity when compared with other approaches of achieving same goal. It is pertinent to mention that whenever $\mathcal{R}_{0}=1$, the disease-free equilibrium is only neutrally stable. However, the application of this algorithm in vector-host models may not be that straight forward as it is in models for one homogeneous population. In such instances, there would obviously be need for additional manipulation of the proposed algorithm in order to obtain the desired result. Worse still, this algorithm does not work for showing global stability of the endemic equilibrium solution. Thus, further research would be necessary in order to extend this algorithm to cover these additional instances.

\section{DECLARATION OF INTERESTS}

The author declare that he has no competing interests that could influence the work reported in this paper.

\section{REFERENCES}

[1] I. S. Jacobs and C. P. Bean, "Fine particles, thin films and exchange anisotropy,' in Magnetism, vol. III, G. T. Rado and H. Suhl, Eds. New York: Academic, 1963, pp. 271-350.

[2] J.K. Asamoah, F. Nyabadza, B. Seidu., M. Chand, and H. Dutta, "Mathematical Modelling of Bacterial Meningitis Transmission Dynamics with Control Measures," Computational and Mathematical Methods in Medicine, 2018, pp.1-21. https://doi.org/10.1155/2018/2657461

[3] S. Ullah and M. A. Khan M.A, "Modeling the impact of nonpharmaceutical interventions on the dynamics of novel coronavirus with optimal control analysis with a case study," Chaos, Solitons and Fractals, vol. 139, 2020, pp. 1-15.

[4] P. Driessche,'Reproduction numbers of infectious diseases," Infectious Diseases Modelling, vol. 2, 2017, pp. 288-303.

[5] A. Elazzouzi1, A. L. Alaoui, M. Tilioua, and A. Tridane, "Global stability analysis for a generalized delayed SIR model with vaccination and treatment," Advances in Difference Equations, vol., 2019, pp. 532: 1-19. https://doi.org/10.1186/s13662-019-2447-z

[6] D. Gao, N. Huang, S. M.Kang, and C. Zang, "Global stability Analysis of an SVIER Epidemic Model with general Incidence Rate," Boundary Value Problems, vol. 42, 2018, pp. 1-22. https://doi.org/10.1186/s13661018-0961-7 
[7] C. O. Ijalana and T. T. Yusuf (2017). "Optimal control strategy for Hepatitis B virus epidemic in areas of high endemicity," International Journal of Scientific and Innovative Mathematical Research, vol. 5(12), 2017, pp. 28-39. http://dx.doi.org/10.20431/2347-3142.0512003

[8] J. LaSalle and S. Lefschetz, "The stability of dynamical systems," SIAM Philadelphia, 1976.

[9] P. Magal, C. C. McCluskey, and G. F. Webb, ” Lyapunov Functional and Global Asymptotic Stability for an Infection-age Model," Applicable Mathematics, vol. 89(7), 2010, pp. $1109-1140$

[10] A. A. Mohsen1, H. F. AL-Husseiny, X. Zhou, and K. Hattaf, "Global stability of COVID-19 model involving the quarantine strategy and media coverage effects," AIMS Public Health, vol. 7(3), 2020, pp. 587605.

[11] Z. Shuai and P. Driessche, "Global Stability of Disease Infectious Models Using Lyapunov Functions," SIAM Journal of Applied Mathematics, vol. 73(4), 2013, pp. 1513 - 1532

[12] T. T. Yusuf and O.Idisi, "Modelling the Transmission Dynamics of HIV and HBV Coepidemics: Analysis and Simulation," Mathematical Theory and Modeling, vol. 10(2), 2020, pp.48-77. 